\title{
Research on the Prediction of Aircraft Landing Distance
}

\author{
Ningning Zhao $\mathbb{D}$ and Junchao Zhang \\ College of Air Traffic Management, Civil Aviation University of China, Tianjin 300300, China \\ Correspondence should be addressed to Ningning Zhao; xianyuer315@163.com
}

Received 22 September 2021; Revised 17 December 2021; Accepted 3 January 2022; Published 20 January 2022

Academic Editor: Francesco Franco

Copyright ( 2022 Ningning Zhao and Junchao Zhang. This is an open access article distributed under the Creative Commons Attribution License, which permits unrestricted use, distribution, and reproduction in any medium, provided the original work is properly cited.

\begin{abstract}
To prevent aircraft from running off the runway during landing, this paper uses a BP neural network model to predict the aircraft landing distance. In this study, based on the five main influencing factors of airport height, aircraft landing quality, airport runway slope, wind, and ambient temperature, the B737-800 was selected as the reference aircraft and the relevant operational data were collected using Boeing's LAND software for the study. In addition, this study uses LM (Levenberg-Marquardt) algorithm and GA (genetic algorithm) to optimize the training process, accelerate the computation speed, and improve the shortage of local optimization of BP (back propagation) neural network model and then construct the GA-LM-BP neural network optimization model. Finally, it makes the BP neural network have the ability of global search for optimal solutions. The results show that the predicted landing data are in good agreement with the measured landing data. The maximum absolute error is within $6.66 \mathrm{~m}$ and the maximum relative error is within $0.038 \%$.
\end{abstract}

\section{Introduction}

Accidents involving aircraft running off the runway during the landing phase occur frequently and can result in injuries and economic losses. According to statistics, there were 626 accidents in civil aviation worldwide from 1959 to 2019. Among them, there were 136 runway accidents, accounting for $21.7 \%$ of the total. In order to ensure the safety of aircraft landing, especially for aircraft operating on wet runways and contaminated runways, the Civil Aviation Administration of China (CAAC) has formulated and issued an advisory circular on "Regulations on the Operation of Wet Runways and Contaminated Runways by Air Carriers." It is clearly stated in the circular that under certain special circumstances (e.g., when airport operating conditions deteriorate), the distance needs to be evaluated before landing [1]. Currently, landing distances are mainly evaluated through performance manuals or performance software provided by the aircraft manufacturer. Among them, the former is more complicated and cumbersome and is not suitable for pilots to use before landing; the latter requires the aircraft to be loaded with relevant performance calculation software such as electronic flight package, which is more restricted in use
[2]. In response to the above problems, Gu and Wang estimated the wet and polluted runway landing distance by using multiple linear regression method. Their derived simplified model algorithm is simple and practical, but the accuracy is relatively low [3]. Xia used the original aerodynamic data and propulsion data of the aircraft to calculate the landing distance of the aircraft. However, due to the extreme confidentiality of these data, the method is applicable to aircraft manufacturers and is more difficult to be applied to the actual operation of airlines [4]. Caiet al. used a support vector machine (SVM) model to predict the aircraft landing distance. They predicted the aircraft landing distance by considering three main factors: airport, weather, and aircraft. However, this prediction accuracy is not high enough [5]. Sun et al. established a mathematical model for landing at highland airports and predicted the landing distance, but the factors considered were relatively single [6]. Fernando et al. linked the similarity theory with a series of factors that can affect aircraft landing and so on to derive a dimensionless equation, while then using real landing data with the regression equation to solve different prediction equations from different pitch angles [7]. However, the factors considered are relatively single and the accuracy of 
the calculation results is not high enough. The researchers at Honeywell, USA, invented and developed a prediction method based on the predicted flight approach slope to determine the effective approach slope of the aircraft and predict the landing distance of the aircraft, which helps to reduce the risk of runway excursion [8]. Qian et al. proposed a landing distance prediction model based on improved extreme learning machine (ELM) with flight data. Firstly, flight data are preprocessed with data slicing method based on flight height and used to determine model input variables. Secondly, a suitable activation function is selected. Subsequently, the PSO-ELM model for landing distance prediction is established. Finally, the conventional BP neural network and ELM are compared under different evaluation indexes. The results show that the prediction of landing distance is consistent with the measured data, but the accuracy of the prediction results is not high enough [9].

The above literature calculates the landing distance of the aircraft from a single angle or with insufficient accuracy. Gao et al. proposed a fetal weight prediction model based on genetic algorithm optimized back propagation (GA-BP) neural network. The computational results showed that the prediction error of GA-BP neural network model was less than $6 \%$, and the accuracy of GA-BPNN was $76.3 \%$, which was $14.5 \%$ higher than the traditional method [10]. Chen et al. proposed a new method called Improved Genetic Algorithm (IGA) Coupled Back-Propagation Neural Network Model (GA-BPNN), which collected meteorological and hydrological data from 2010 to 2017 for experiments. The results show that the GA-BPNN model can solve the problems of limited optimization effect and local convergence, while improving the prediction accuracy and model stability [11]. Yao proposed the ABC-based GA$\mathrm{NN}$ model and applied it to the civil aircraft customer service cost prediction method. Experiments proved that the ABC-based GA-NN model can not only bring into play the powerful search capability of GA algorithm, but also outperforms the traditional NN network model in terms of prediction accuracy and convergence speed [12]. Ma et al. use genetic algorithms to optimize the neural network model built to further improve the network prediction performance. The data calculation results showed that the method exhibited higher accuracy and stability in airport aviation noise prediction [13]. Liu et al. use the BP neural network improved by the LM algorithm to obtain the tunnel displacement back analysis model, and the calculation results show that the accuracy is as high as $97.3 \%$ [14]. Zhang et al. designed and applied a numerical optimization algorithm based on LM-based BP neural network model to predict the line loss rate of each transformer area data category. The method verifies the accuracy of the proposed prediction method using 1026 transformer zones with complete power operation data in a region [15]. Some scholars have combined LM and GA algorithms to optimize the BP neural network model and have achieved good experimental results. For example, Wang's introduction of LM into BP neural network can solve local minimum problems and speed up the convergence speed. The optimized genetic LM-BP algorithm is applied to the optimal design of FIR digital filters. By comparing with the filter designed by classical BP neural network, the filter designed by the optimized algorithm achieves good optimal design results [16]. Yao used LM algorithm to improve the training algorithm. He used genetic algorithm to optimize the initial weights and thresholds to obtain the improved GA-LM-BP neural network. The improved model is used for urban rail transit short-time passenger flow prediction. The obtained results show that the improved model greatly improves the efficiency and accuracy of prediction compared with the traditional BP neural network [17]. However, the combination of LM and GA for neural network model optimization has not been sufficiently studied. Therefore, in order to achieve faster data processing speed and high accuracy prediction results, an optimized neural network model combining LM and GA algorithms is used in this study. Meanwhile, this study integrates five factors: airport height, aircraft landing quality, airport runway slope, wind, and ambient temperature to predict aircraft landing distance with high accuracy.

\section{Optimized BP Neural Network Prediction Model}

2.1. BP Neural Network Model. There are two main processes in BP neural network models, namely training and testing. The training is especially important, which includes the calculation process in the forward direction and the calculation process in the reverse direction, which is related to the good or bad results obtained. After the training, a nonlinear complex mapping model is obtained. The overall model process is completed by substituting the test landing distance data and performing a landing distance model test. And a typical model has three layers, input, output, and hidden layers, and consists of nodes in each layer. Except for the nodes juxtaposed in the same layer, the nodes in adjacent layers are connected enough through weights with a series of operational formulas. Where the training process algorithm flows, the three layers of the model according to the input process set three nodes, $m, p, q$; input data (landing conditions) $\mathbf{x}=\left(x_{1}, x_{2}, \ldots, x_{m}\right)$; input to the hidden layer data $\mathbf{s}_{\mathbf{i}}=\left(s i_{1}, s i_{2}, \ldots, s i_{p}\right)$; the hidden layer output data $\mathbf{s}_{\mathbf{j}}=$ $\left(s j_{1}, s j_{2}, \ldots, s j_{p}\right)$; input to the output layer data $\mathbf{b}_{\mathbf{i}}=\left(b i_{1}, b\right.$ $\left.i_{2}, \ldots, b i_{q}\right)$; the output layer output data $\mathbf{b}_{\mathbf{j}}=$ $\left(b j_{1}, b j_{2}, \ldots, b j_{q}\right)$; the actual output of the landing distance data $\mathbf{t}_{\mathbf{j}}=\left(t_{1}, t_{2}, \ldots, t_{q}\right)$. The weight value $w_{i s}$ is the weight between the hidden layer and the input layer, $w_{s j}$ is the weight between the hidden layer and the output layer, $d_{r}$ is the threshold for each node in the hidden layer; $d_{j}$ is the threshold for the node in the third layer; and the amount of sample data selected are as follows: $\theta=1, \ldots, n$. Let the error function be (1), and the transfer function in the latter two layers will come out with $f_{1}(x)$ and $f_{2}(x)$. Set the input sample of condition-specific data for landing as $\mathbf{x}(\theta)=\left(x_{1}\right.$ $\left.(\theta), x_{2}(\theta), \ldots, x_{m}(\theta)\right)$, and set the actual output specific aircraft landing distance data $\mathbf{t}(\theta)=\left(t_{1}(\theta), t_{2}(\theta)\right.$, $\left.\ldots, t_{m}(\theta)\right)$. 


$$
E=\frac{1}{2} \sum_{j=1}^{q}\left(t_{j}(\theta)-b_{j}(\theta)\right)^{2} .
$$

$\eta$ is the learning rate. The learning rate is the amount of weight updates during training which can adjust the rate of weight changes. Since the learning rate is a visual representation of the weight change, it is often used to control the weight change. The specific value of the learning rate often ranges from 0 to 1 . A higher learning rate results in faster weights and vice versa. When the learning rate is too large, the amount of correction is too large. Sometimes the learning rate can exceed the error value and cause jumps. Too small a learning rate makes the training time too long. For a normal model, a learning rate of some number between 0.01 and 0.1 is better.

Positive propagation of input information:

$$
\begin{aligned}
& s i_{r}(\theta)=\sum_{i=1}^{m} w_{i s} x_{i}(\theta)+d_{r}, \\
& s j_{r}(\theta)=f_{1}\left(s i_{r}(\theta)\right), \\
& b i_{j}(\theta)=\sum_{j=1}^{p} w_{s j} s j_{r}(\theta)+d_{j}, \\
& b j_{j}(\theta)=f_{2}\left(b i_{j}(\theta)\right),
\end{aligned}
$$

where $r=1,2, \ldots, p, j=1,2, \ldots, q$.

Back propagation of error information:

update of the $N+1$ generation hidden layer with previously connected weight value $w_{s j}$ :

$$
\mathrm{w}_{s j}^{N+1}=w_{s j}^{N}+\eta \cdot \alpha_{j}(\theta) \cdot s j_{r}(\theta) .
$$

Update of the $N+1$ generation output layer threshold:

$$
d_{j}^{N+1}=d_{j}^{N}+\eta \cdot \alpha_{j}(\theta) .
$$

Update of the $N+1$ generation hidden layer with the weight value $w_{i s}$ connected thereafter:

$$
w_{i s}^{N+1}=w_{i s}^{N}+\eta \cdot \alpha_{i}(\theta) \cdot x_{i}(\theta) .
$$

Update of the $N+1$ generation hidden layer weights:

$$
d_{r}^{N+1}=d_{r}^{N}+\eta \cdot \alpha_{i}(\theta)
$$

In this paper, the functions set in the hidden and output layers of the model are different, respectively, the tanh function whose derivative is as in (7) and the linear function whose derivative is 1 , which can be obtained by (6).

Order:

$$
e_{j}=t_{j}(\theta)-b_{j}(\theta)
$$

From

$$
\begin{aligned}
\tanh ^{\prime}(x) & =\left(\frac{\sinh (x)}{\cosh (x)}\right)^{\prime} \\
& =1-\tanh ^{2}(x) .
\end{aligned}
$$

This ultimately leads to

$$
\begin{aligned}
& w_{s j}^{N+1}=w_{s j}^{N}+\eta \cdot e_{j} \cdot s j_{r}(\theta), \\
& d_{j}^{N+1}=d_{j}^{N}+\eta \cdot e_{j}, \\
& w_{i s}^{N+1}=w_{i s}^{N}+\eta \cdot \sum_{j=1}^{q}\left(e_{j} \cdot w_{s j}^{N}\right) \cdot\left(1-s j_{r}^{2}(\theta)\right) \cdot x_{i}(\theta), \\
& d_{r}^{N+1}=d_{r}^{N}+\eta \cdot \sum_{j=1}^{q}\left(e_{j} \cdot w_{s j}^{N}\right) \cdot\left(1-s j_{r}^{2}(\theta)\right) .
\end{aligned}
$$

2.2. BP Neural Network Optimization Algorithm. The LM algorithm and genetic algorithm are used to optimize the training process to construct the LM-BP neural network model, which makes the training operation speed up and solves the shortcomings of local optimization of BP neural network model at the same time. The genetic algorithm optimizes the initial weight threshold to construct the GALM-BP neural network model, which makes the BP neural network have the ability of global search for optimal solutions.

The fitness function of the genetic algorithm is

$$
\begin{aligned}
k & =\frac{1}{e} \\
& =\frac{1}{\operatorname{norm}\left(t_{j}-d_{j}, \text { inf }\right)},
\end{aligned}
$$

where norm denotes the computed parametric number, $t$ is the expected value, $e$ denotes the computed vector parametric number, $d$ is the predicted value, and inf denotes the maximum value of the absolute value in the computed vector.

Using the probability fitness obtained by the formula, select individuals for inheritance, and the calculation formula for the fitness value is shown in

$$
P_{j}=\frac{k_{j}}{\sum_{m=1}^{n} k_{m}} .
$$

The crossover probabilities are generally chosen to be large to enhance the algorithm rate and further improve the speed of GA operations. However, when the probability is too large, it will cause the evolutionary law to fail and also make the search randomized. If the probability is too small, the algorithm rate decreases but also improves the global search. It can be seen that it is better to use a probability within 0.4 to 0.99 . Therefore, in this paper, 0.7 is used as the probability. The variation probability is often within 0.001 to 0.1 . As with biological inheritance, the probability of genetic variation is small. Therefore, in this paper, 0.01 is chosen as the variation probability.

Taken together, some of the parameters of the genetic algorithm are set as Table 1 .

The optimization algorithm flow is shown in Figure 1, where the BP neural network algorithm part is inserted into the genetic algorithm part as a function to calculate the value of the assigned fitness, and finally the output weight threshold is saved for the model training and then can be tested several times. 
TABLE 1: Genetic algorithm parameters.

\begin{tabular}{lc}
\hline Models & Parameters \\
\hline Number of groups & 40 \\
Coding method & Binary encoding \\
Crossover probability & 0.7 \\
Mutation probability & 0.01 \\
Maximum number of genetic generations & 50 \\
\hline
\end{tabular}

2.3. Evaluation Indicators. The evaluation index is calculated by the following formula, which is used to measure the RootMean-Square Error (RMSE), Average Relative Error (MRE), Mean Absolute Error (MAE), and Mean Squared Error (MSE) of the prediction accuracy.

$$
\begin{aligned}
& E_{\mathrm{RMSE}}=\sqrt{\frac{1}{M} \sum_{a=1}^{M}\left(x_{a}-y_{a}\right)^{2},} \\
& E_{\mathrm{MRE}}=\frac{1}{M} \sum_{a=1}^{M}\left|\frac{x_{\mathrm{a}}-y_{a}}{y_{a}}\right|, \\
& E_{\mathrm{MAE}}=\frac{1}{M} \sum_{a=1}^{M}\left|x_{\mathrm{a}}-y_{a}\right|, \\
& E_{\mathrm{MSE}}=\frac{1}{M} \sum_{a=1}^{M}\left(x_{a}-y_{a}\right)^{2} .
\end{aligned}
$$

\section{Application Analysis}

3.1. Data Collection and Preprocessing. For a flight, if the condition of the landing runway surface has been specified, it can be considered that the factors changing the landing distance are mainly airport altitude, aircraft landing quality, airport runway slope, wind, and ambient temperature [4]. In this paper, these five factors are selected as input vectors and the landing distance is the output vector. In this paper, we collected the relevant data according to Boeing performance software and chose B737-800 as the predicted model, as well as selecting the dry runway as the landing runway condition. The $V_{\text {REF }}$ was set to runway entrance speed, and the ground spoiler was set to automatic; the aircraft configuration was the most commonly used landing configuration for landing, the air conditioner was turned on, and the flap position was $15^{\circ} / 30^{\circ}$, which was the position used for approach and landing respectively. Since China uses manual landing for landing operation, the landing condition is manual landing and the braking method also uses manual. Moreover, the auxiliary power unit is off; the tire speed is $225 \mathrm{mph}$; the antistalling part is set to effective and the anti-icing part is set to off. 700 data were selected for data analysis by the above conditions, using 570 of them for the training process and 130 for the testing process. Some of the raw data are shown in Tables 2 and 3 (due to the large amount of data, only 30 sets of data are presented. Among them, 15 sets of data are used for training and 15 sets of data are used for testing. These 30 sets of data are randomly selected).
Specifically in Table 2, $V$ represents the wind speed in $\mathrm{kt}$ and sets the downwind speed as a negative number, $T$ represents the temperature of the environment in ${ }^{\circ} \mathrm{C}, W$ represents the landing mass in $\mathrm{kg}, H$ represents the airport height in $\mathrm{m}, I$ represents the runway slope in $\%$, and $Y$ represents the required runway distance in $\mathrm{m}$.

In this paper, the tanh function is chosen as the activation function of the hidden layer. This function is smooth, continuous, and differentiable. Moreover, it has a wider change-sensitive interval than the sigmoid function and a more delayed saturation period than the sigmoid function. It performs a normalization operation on the data to be substituted, mapping each data value to between -1 and 1 . This treatment not only eliminates the inconsistent effect of the magnitude and makes the mathematics pure, but also improves the convergence rate of the model and protects the landing distance model from the negative effect of saturation. Meanwhile, since the hidden layer transfer function in this paper is a tanh function, the input value has a high sensitivity at $[-1,1]$. If the input value exceeds this range, the function will enter a saturation state, which will further affect the accuracy of the error data values. For this reason, when substituting data into the neural network model for calculation, the data to be substituted is performed with a normalization operation that maps each data value to between -1 and 1 . The normalization formula is

$$
y=\frac{\left(y_{\max }-y_{\min }\right) \cdot\left(x-x_{\min }\right)}{x_{\max }-x_{\min }}+y_{\text {min }},
$$

where $x$ is the input value, $y_{\max }$ is the specific number 1 (maximum value), $y_{\text {min }}$ is the specific number -1 (minimum value), and the function $y$ is the number of substitution used in this paper and the $x$-normalized number.

\subsection{Construction of the Landing Distance Prediction Model} Based on BP Neural Network. The selection of the number of neurons in the hidden layer is critical to the construction of the neural network. Choosing too many will consume too much time and may even lead to too severe fitting. Too little number of neurons will make the prediction accuracy too low. Therefore, it is necessary to use the empirical formula (equation (14)) first and then conduct repeated experiments to get the number of neurons.

$$
g=\sqrt{r+j}+l
$$

where $g$ is the number of nodes in the hidden layer, $r$ is the number of terms in the condition, $j$ is the number of output terms (for the landing distance) is 1 , and $l$ denotes a constant between 1 and 10 .

A review of the literature shows that the performance of the prediction is better when the model uses multiple hidden layers to train the network. The neurons of the hidden layers in the model are determined by the minimum mean square error values obtained during the study of the network parameters. In the case of this paper, the number of hidden layer nodes can be chosen between 3 and 12. In this study, the number of hidden layer nodes between 3 and 12 is calculated several times using the enumeration method, and then their root mean square 


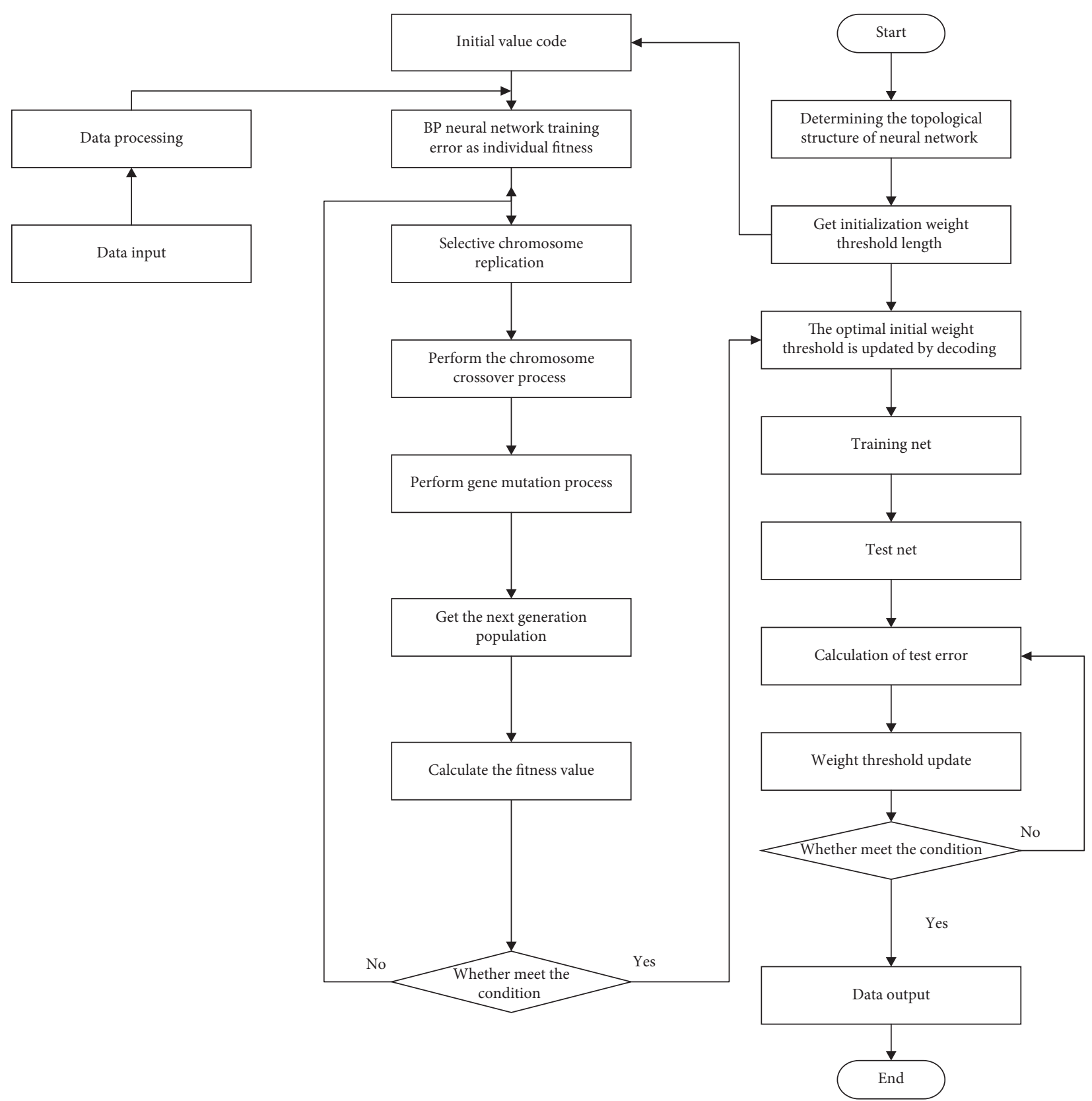

FIgURE 1: Improved model flow chart.

Table 2: Training data for landing distance of B737-800 (partial).

\begin{tabular}{|c|c|c|c|c|c|c|}
\hline Training & $V / \mathrm{kt}$ & $T /{ }^{\circ} \mathrm{C}$ & $W / \mathrm{kg}$ & $H / \mathrm{m}$ & $I / \%$ & $S / \mathrm{m}$ \\
\hline 1 & -7.7 & -21 & 64500 & 2250 & 0.09 & 2279 \\
\hline 2 & -6.1 & -31 & 58200 & 3300 & -0.13 & 2191 \\
\hline 3 & 3 & 7 & 61200 & 643 & 0.07 & 1690 \\
\hline 4 & -10 & 16 & 53800 & 3600 & -0.03 & 2188 \\
\hline 5 & -7.3 & 12 & 63000 & 88 & -0.01 & 1884 \\
\hline 6 & -6 & -16 & 56500 & 3600 & 0.07 & 2179 \\
\hline 7 & 4.4 & 8 & 48800 & 2530 & -0.03 & 1576 \\
\hline 8 & 3.1 & 9 & 57200 & 3300 & -0.13 & 1959 \\
\hline 9 & 7 & 27 & 55400 & 3000 & -0.15 & 1817 \\
\hline
\end{tabular}


TABLE 2: Continued.

\begin{tabular}{lcccccc}
\hline Training & $V / \mathrm{kt}$ & $T /{ }^{\circ} \mathrm{C}$ & $W / \mathrm{kg}$ & $H / \mathrm{m}$ & $I / \%$ & -1.04 \\
\hline 10 & 1.5 & -3 & 60100 & 1560 & 952 & 1799 \\
11 & -0.9 & -10 & 65800 & 952 & -19 & 1914 \\
12 & -6.9 & -10 & 65800 & 3000 & -0.11 & 2079 \\
13 & 1 & -27 & 59600 & 3000 & 2011 \\
14 & 0 & -15 & 59500 & 3000 & 0.07 & -0.03 \\
15 & 3 & -15 & 59500 & & 1990 \\
\hline
\end{tabular}

TABLE 3: Selected test data of the landing distance of the B737-800 (partial).

\begin{tabular}{|c|c|c|c|c|c|c|}
\hline Testing & $V / \mathrm{kt}$ & $T /{ }^{\circ} \mathrm{C}$ & $W / \mathrm{kg}$ & $\mathrm{H} / \mathrm{m}$ & $I / \%$ & $S / \mathrm{m}$ \\
\hline 1 & 6.9 & -10 & 65800 & 952 & -0.06 & 1829 \\
\hline 2 & -9.1 & -16 & 58000 & 3300 & -0.13 & 2270 \\
\hline 3 & 0 & -3 & 59100 & 1560 & -1.11 & 1783 \\
\hline 4 & 2.2 & -16 & 60300 & 2880 & -0.01 & 2003 \\
\hline 5 & -7.4 & 8 & 42300 & 2530 & 0.09 & 1617 \\
\hline 6 & -8.9 & -10 & 65800 & 952 & 0.31 & 2136 \\
\hline 7 & 0.6 & -10 & 63100 & 2060 & -0.02 & 1976 \\
\hline 8 & 7.7 & -17 & 64500 & 2250 & -0.03 & 1987 \\
\hline 9 & 3.1 & 15 & 56100 & 3300 & -0.01 & 1923 \\
\hline 10 & 0 & -16 & 56500 & 3600 & 0.07 & 2014 \\
\hline 11 & 2.1 & 7 & 57600 & 3300 & 0.15 & 1982 \\
\hline 12 & 7 & 29 & 54500 & 3000 & -1.1 & 1788 \\
\hline 13 & 10 & 17 & 53600 & 3600 & 0.15 & 1823 \\
\hline 14 & 0.6 & -10 & 41900 & 2060 & -0.11 & 1376 \\
\hline 15 & 0 & 31 & 51400 & 3300 & -0.01 & 2267 \\
\hline
\end{tabular}

TABLE 4: The selected neurons and their corresponding RMSE.

\begin{tabular}{lc}
\hline Number of hidden layer neurons & RMSE \\
\hline 3 & 48.12156 \\
4 & 46.14444 \\
5 & 47.66138 \\
6 & 47.53927 \\
7 & 45.7321 \\
8 & 47.52606 \\
9 & 46.26877 \\
10 & 46.09896 \\
11 & 46.8748 \\
12 & 44.29004 \\
\hline
\end{tabular}

error is obtained. The results are shown in Table 4. It is known that the more the RMSE is close to zero, the better the prediction model is obtained. From the results in Table 4, it can be seen that the lowest value of RMSE is obtained when the number of neurons in the hidden layer is 12 . Therefore, the model with the number of hidden layer nodes of 12 is chosen in this paper, which has the best performance and better prediction model accuracy.

From that when the number of hidden layer neurons is 12 , the RMSE value is the lowest, the model performance is optimal, and the prediction model accuracy is better, so the number of hidden layer nodes 12 is chosen for the model.

The constructed BP neural network based landing distance prediction model is shown in Figure 2.

The specific parameters of the designed model are shown in Table 5.
3.3. Analysis of Prediction Results of the Ordinary BP Neural Network Model. It can be seen from Table 6 that the common BP neural network model has too much error in predicting the aircraft landing distance, and the model needs to be improved and optimized to improve the accuracy of the prediction.

3.4. Analysis of Prediction Results. The aircraft landing distance prediction study was carried out by GA-LM-BP model, the comparison of the obtained prediction results with the actual values is shown in Figure 3, their accuracy is shown in Table 7, and the comparison of the accuracy of the optimized model with the unoptimized one is shown in Table 8.

As can be seen from Figure 3, the highest prediction accuracy is achieved around the 38th generation, and its initial weight threshold is taken for the prediction study.

In this study, the GA-LM-BP model was used for prediction study of aircraft landing distance. Figure 4 shows the comparison between the predicted and actual values of the GA-LM-BP model. From the figure, it can be seen that the error between the predicted data and the actual data is low and in better agreement. And the maximum error is only within the range of $6.66 \mathrm{~m}$. Figure 5 shows the absolute errors of the predictions. Figure 6 shows the relative errors of the predictions. As can be seen from the figures, the maximum relative error is only about $0.038 \%$, and the prediction is in good agreement with the actual one. In summary, it is feasible to use the GA-LM-BP model to predict the aircraft landing distance for the study. 


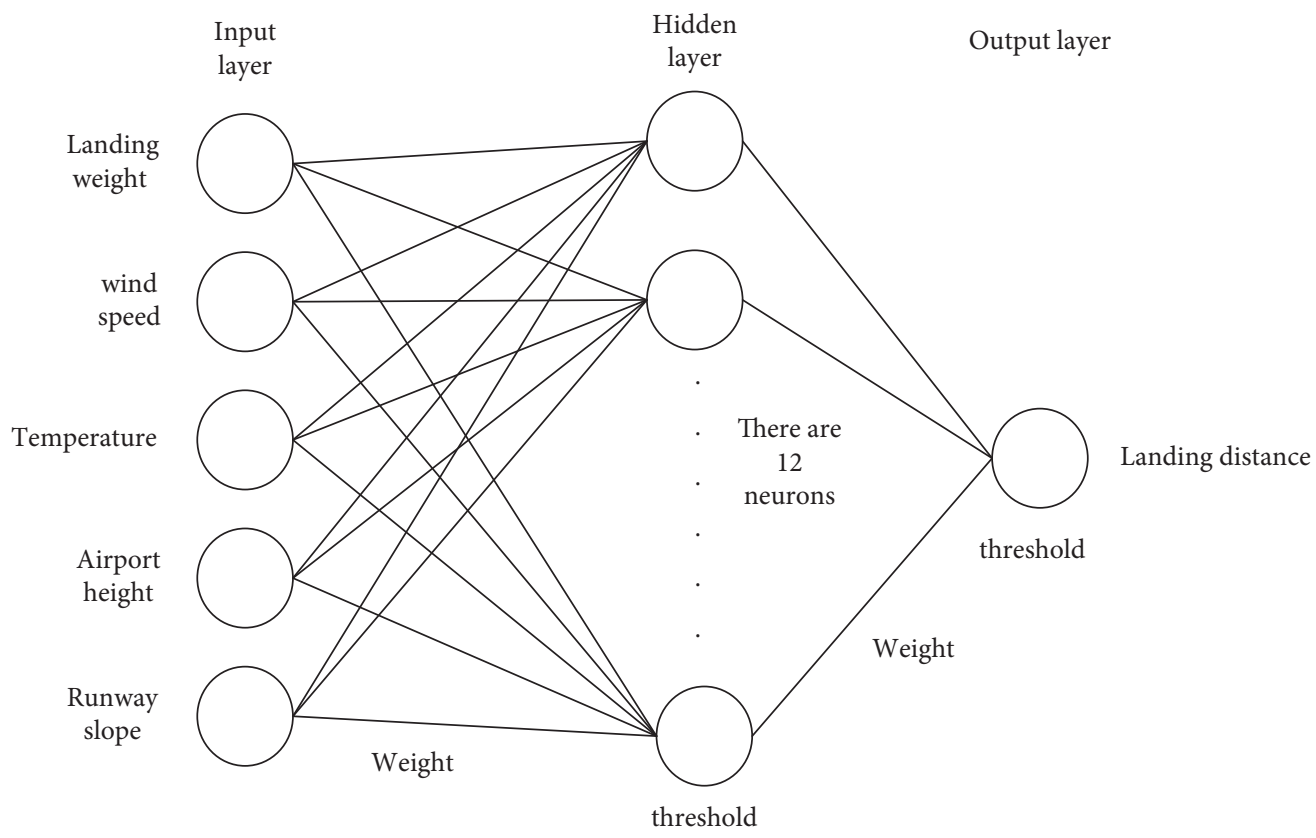

FIgURE 2: Structure diagram of the BP neural network model.

TABLE 5: BP neural network model.

\begin{tabular}{lc}
\hline Structure & Parameters \\
\hline Hidden layer activation function & tanh \\
Output layer activation function & Purelin \\
Learning rate & 0.01 \\
Training algorithms & Gradient descent method \\
Number of hidden layers & 1 \\
Number of hidden layer neurons & 12 \\
Number of output layer layers & 1 \\
Number of neurons in the output layer & 1 \\
Number of input layer neurons & 5 \\
\hline
\end{tabular}

TABLE 6: Neural network model accuracy evaluation value.

\begin{tabular}{lc}
\hline Rate the value & General BP neural network \\
\hline$E_{\mathrm{MAE}}$ & 16.31568 \\
$E_{\mathrm{MRE}}$ & $0.8843 \%$ \\
$E_{\mathrm{MSE}}$ & 44.29004 \\
Maximum absolute error & 59.4255 \\
Maximum relative error & $4.165 \%$ \\
\hline
\end{tabular}




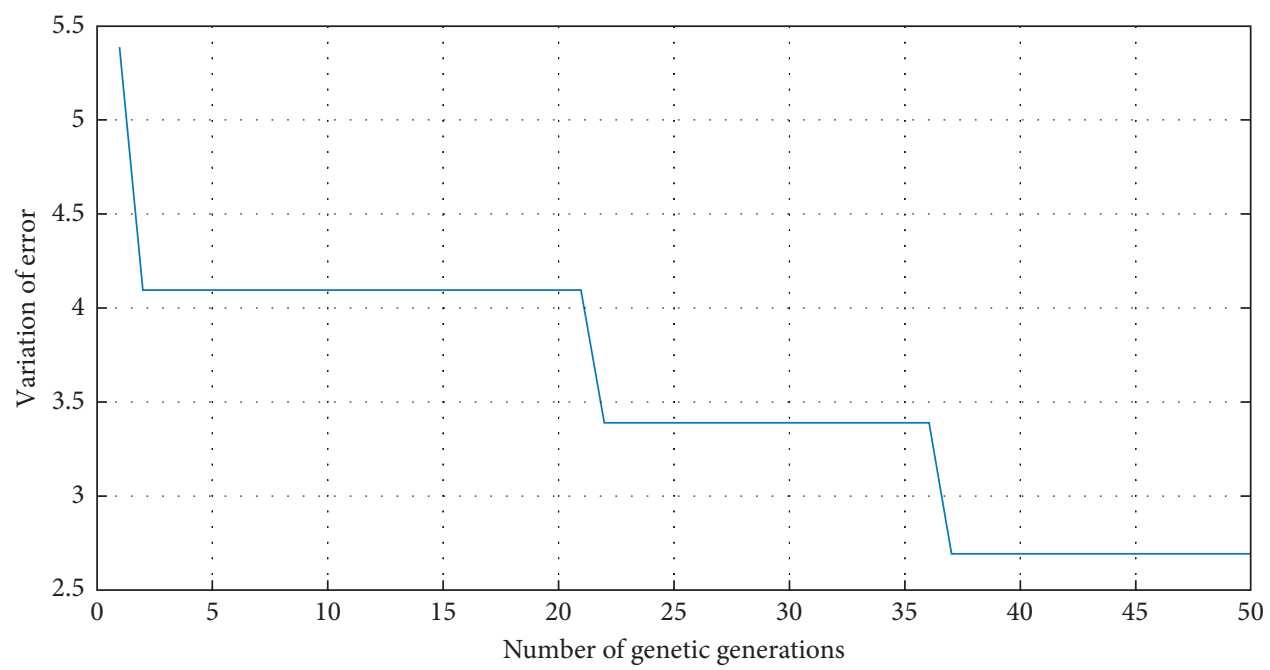

Figure 3: The evolution diagram of prediction error.

TABLE 7: Evaluation value of GA-LM-BP model prediction accuracy.

Rate the value

$E_{\mathrm{MAE}}$

$E_{\mathrm{MRE}}$

$E_{\mathrm{MSE}}$

Maximum absolute error

Maximum relative error
GA-LM-BP neural network

1.315094

$0.0727 \%$

3.169887

6.657827

$0.03757 \%$

TABLE 8: Evaluation indexes of each model.

\begin{tabular}{|c|c|c|c|c|c|}
\hline Rate the value & MAE & MRE (\%) & $\mathrm{MAE}_{\max }$ & $\mathrm{MRE}_{\max }(\%)$ & MSE \\
\hline $\mathrm{BP}$ & 16.31568 & 0.8843 & 59.4255 & 4.165 & 44.29004 \\
\hline LM-BP & 1.764987 & 0.0975 & 7.568207 & 0.446 & 5.84701 \\
\hline GA-LM-BP & 1.315094 & 0.0727 & 6.657827 & 0.03757 & 3.169887 \\
\hline
\end{tabular}

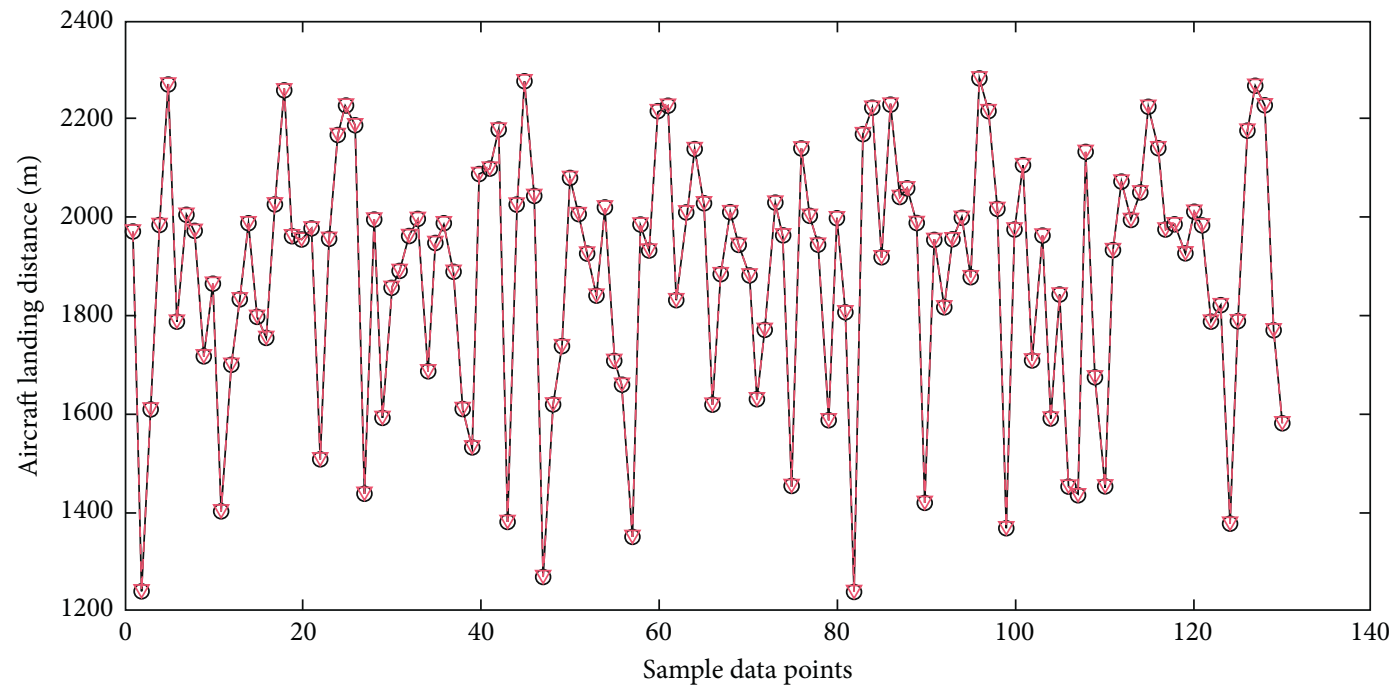

$\odot$ Original data

$-\nabla-$ Prediction data

FIgURE 4: Comparison of predicted and actual values of landing distance. 


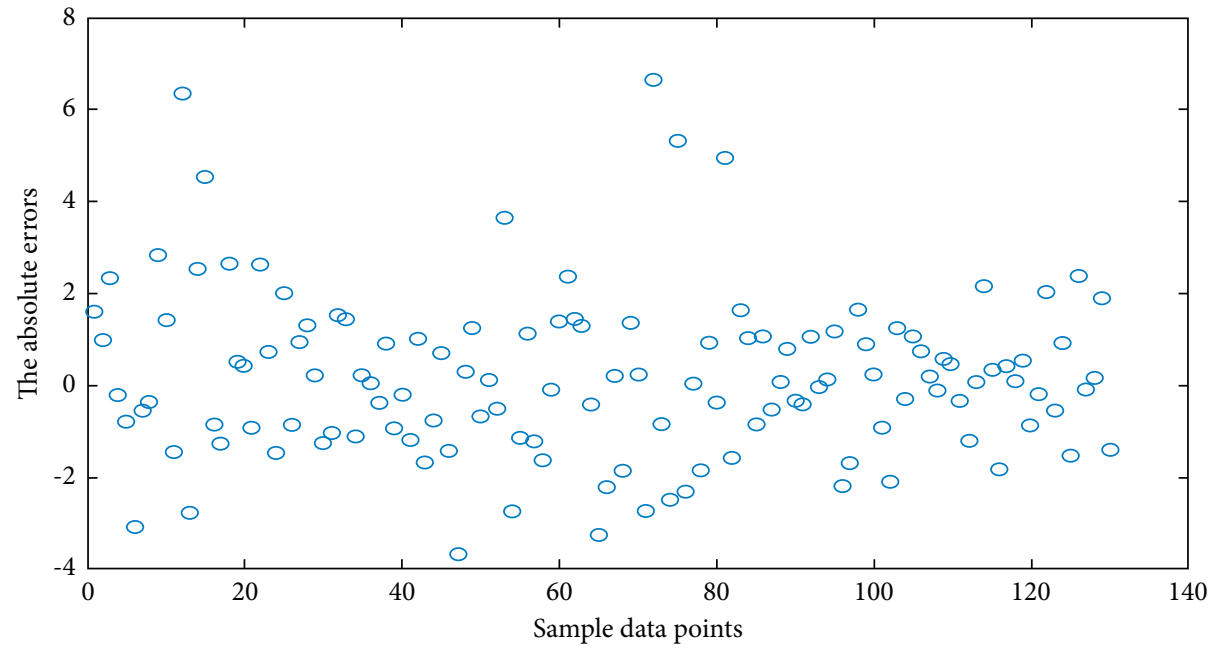

FIgURE 5: The absolute errors of prediction results.

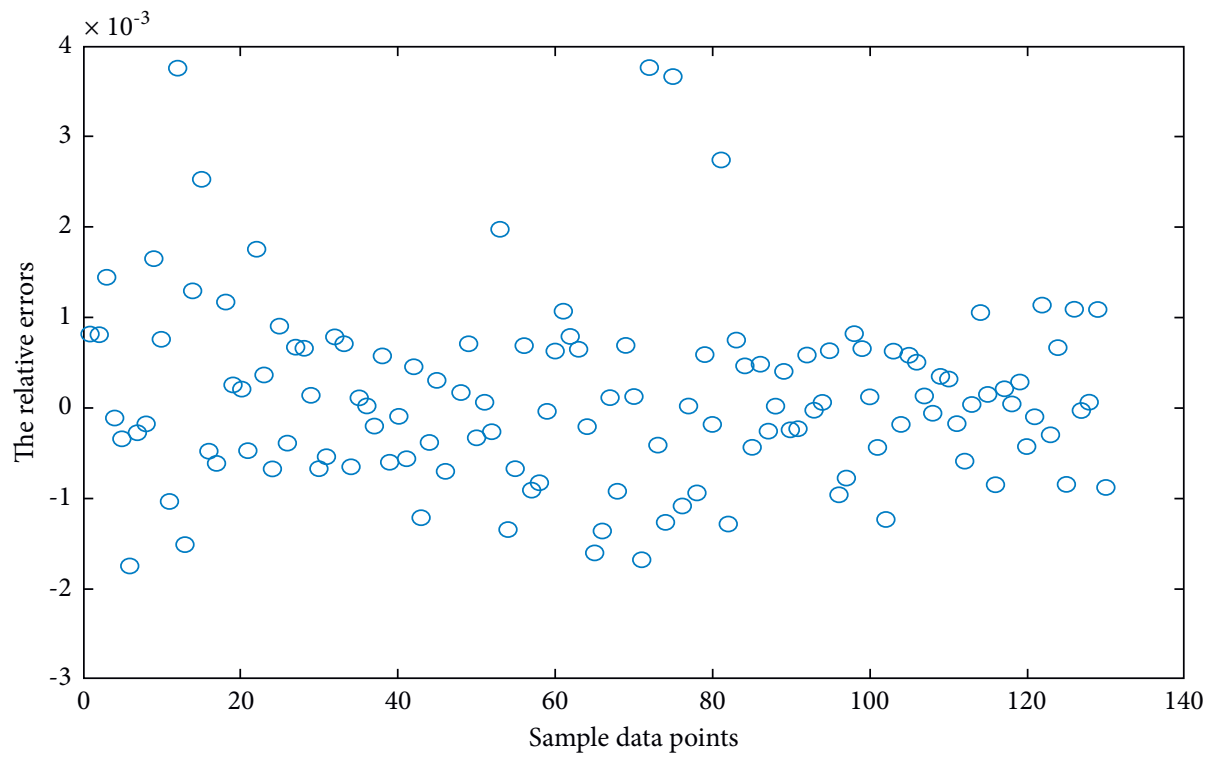

FIGURE 6: The relative errors of prediction results.

\section{Conclusion}

The following conclusions were drawn from the study of the paper:

(1) Using Boeing performance software and a specific model B-737-800 as the reference model, the required data were collected and processed by normalization method. Then, the data of five different landing conditions are determined as input variables, the aircraft landing distance is the output variable, and the hidden layer is 12 . This study establishes the aircraft landing distance prediction model based on BP neural network by the above method.

(2) The GA algorithm is used to determine the initial weight threshold to reduce the prediction error so that the model can achieve the effect of global search. In addition, the LM algorithm is used as the training algorithm to improve the convergence and computational speed of the training data. Based on this, a BP neural network model based on GA-LM is constructed to predict the aircraft landing distance.

(3) Based on the actual operational data, the constructed GA-LM-BP model is used to predict the aircraft landing distance. Comparing the actual values with the predicted values, it can be seen that the maximum absolute error is within $6.66 \mathrm{~m}$ and the maximum relative error is within $0.038 \%$. The calculation results of the algorithm show that the predicted values of the aircraft landing distance match with the actual values, and the accuracy of the calculation results is high.

Shortcomings of the study: this paper focuses on the prediction of aircraft landing distance based on the main influencing factors (e.g., landing weight, airport runway 
slope, wind, ambient temperature, and airport altitude) that affect aircraft landing. Although the model constructed in this paper can also be applied to other runway conditions (e.g., wet polluted runways), this paper only introduces data for dry runways in the landing conditions and not for wet polluted runways. In the future, specific landing scenarios and parameters of wet contaminated runways can be considered.

In summary, the GA-LM-BP neural network model constructed in this paper can be used for the prediction of civil aircraft landing distance. The model is more comprehensive in considering the influencing factors, and the calculation results are more accurate and can be applied in practice [18].

\section{Data Availability}

The first part of the data is 700 groups of landing distance data obtained through random conditions, including temperature, runway slope, airport elevation, wind speed, and landing weight. The data are randomly disturbed. The first 570 groups are used for training and then 130 groups are used for testing. The second part of the data is the prediction data obtained by using the number of neurons in different hidden layers, in which the number of neurons ranges from 3 to 12 . The third part is the landing distance predicted by BP neural network model optimized by LM algorithm, which has been predicted for 15 times. The fourth part is the landing distance predicted by GA-LM-BP model, which has been predicted for 15 times.

\section{Conflicts of Interest}

The authors declare that they have no conflicts of interest.

\section{Acknowledgments}

This study was funded by the National Key R\&D Project, "Accurate Awareness of the Activity Situation in the Flight Area and Risk Identification Technology" (2020YHB1600101), National Natural Science Foundation of China, "Research on Dynamic Evaluation and Short-Term Forecast of Flight Operation Risk Accuracy" (U1933103), and the Central University's Fundamental Scientific Research Funds Project, specially funded by the Civil Aviation University of China (3122020050).

\section{References}

[1] Civil Aviation Administration of China, Ac-121-Fs-2009-33, Operational Management Rules on the Wet Runway and the Contaminated Runway of the Air Carrier.

[2] Ry Wen, B. Wu, S. L. Chu, and H. Y. Wang, "Research on landing distance prediction for civil aircraft," Chinese Journal of Safety Science, vol. 27, no. 1, pp. 77-81, 2017.

[3] R. P. Gu and P. Wang, "Estimation of wet/contaminated runway landing distance based on multiple linear regression," Journal of Civel Aviation University of China, vol. 32, no. 3, pp. 20-22, 2014.
[4] J. Xia, Study on Algorithm of Aircraft landing Distance and Example Analysis, Civil Aviation Flight University of China, Deyang, China, 2016.

[5] L C. Cai, Y. Z. Zhang, and H. F Wang, "Calculation and analysis of plane's landing slipping length with full thrust in plateau air field Journal of Air Force Engineering University," Nature Science Edition, vol. 15, no. 3, pp. 5-9, 2014.

[6] R. S. Sun, X. Chen, and C. F. Li, "Prediction method of actual landing distance of aircraft based on similar theory," Chinese Journal of Safety Science, vol. 31, no. 3, pp. 13-18, 2021.

[7] C. A. Fernando, J. P. Carlos, and S. A. Eduardo, "A bayesiannetwork-based approach to risk analysis in runway excursions," Journal of Navigation, vol. 72, no. 5, pp. 1-19, 2019.

[8] Honeywell International Inc, "Method and system for determining effective approach angle and predicting aircraft landing distance," Honeywell International Inc., Charlotte, NC, USA, USPTO 20180102059 [P], 2018.

[9] S. L. Qian, S. H. Zhou, W. B. Chang, and F. J. Wei, “An improved aircraft landing distance prediction model based on particle swarm optimization-extreme learning machine method," in Proceedings of the IEEE International Conference on Industrial Engineering and Engineering Management, pp. 2326-2330, IEEE, Singapore, December 2017.

[10] H. Gao, C. Y. Wu, C. Wu, D. Huang, and D. Zha, "Prediction of fetal weight based on back propagation neural network optimized by genetic algorithm," Mathematical Biosciences and Engineering, vol. 18, no. 4, pp. 4402-4410, 2021.

[11] N. C. Chen, C. Xiong, W. Y. Du, C. Wang, X. Lin, and Z. Q. Chen, "An improved genetic algorithm coupling a backpropagation neural network model (IGA-BPNN) for waterlevel predictions," vol. 11, no. 9, p. 1795, 2019.

[12] J. F. Yao, The Application of the ABC-Based GA-NN Model in Aircraft Customer Service Cost Prediction, Nanjing University of Aeronautics and Astronautics, Nanjing, China.

[13] L. N. Ma, Y. Tian, and X. Y. Wu, "Airport aviation noise prediction based on an optimized neural network," Transactions of Nan Jing University of Aeronautics \& Astronautics, vol. 38, pp. 32-39, 2021.

[14] C. Liu, Y. S. Zhou, H. Z. Liu, and H. G. Zhang, "Research on back analysis of tunnel displacement based on improved BP neural network," Highways, vol. 66, no. 9, pp. 385-392, 2021.

[15] Y. M. Zhang, G. H. Yang, X. Y. Hao, and S. Yao, "Research on prediction model of line loss rate in transformer district based on LM numerical optimization and BP neural network," IOP Conference Series: Earth and Environmental Science, vol. 252, no. 3, Article ID 032095, 2019.

[16] Y. P. Wang, Application of BP Neural Network Based on Genetic Algorithm and LM Algorithm to the Design of Digital Filter, Nanjing University of Aeronautics and Astronautics, Nanjing, China, 2008.

[17] F. Yao, "Research on short-term passenger flow forecast of urban rail transit based on improved Ga-LM-BP neural network," in Proceedings of the World Forum on Transportation Engineering Technology (WTC2021) (Part1), People's Communications Publishing House Co., Ltd., Xian, China, May 2021.

[18] L. Wang and Y. Liang, "Statistics and analysis of global civil aviation accident investigation data," Comprehensive Transportation, vol. 43, no. 3, pp. 7-12, 2021. 